\title{
Effect of Hospital to Home nutrition management model on postoperative clinical outcomes of patients with laryngeal carcinoma
}

\author{
GUIHUA SONG and HAIZHEN LIU \\ Department of Clinical Nutrition, Weifang People's Hospital, Weifang, Shandong 261041, P.R. China
}

Received May 26, 2017; Accepted July 21, 2017

DOI: $10.3892 / \mathrm{ol} .2017 .6709$

\begin{abstract}
The purpose of this investigation was to determine the effectiveness of a Hospital to Home $(\mathrm{H} 2 \mathrm{H})$ nutrition management model on the postoperative clinical outcomes of patients with laryngeal carcinoma. Eighty-six patients with laryngeal carcinoma were randomly divided into an observation ( $\mathrm{H} 2 \mathrm{H}$ nutrition management) or control group (routine nutrition management). The ratios of urine lactulose to mannitol $(\mathrm{L} / \mathrm{M})$ in serum orexin-A levels of patients in both groups on the 1st postoperative day were significantly higher than before surgery; these levels decreased from the 7th and 14th postoperative day, but remained higher than before surgery $(p<0.05)$. There was no statistical difference in the $\mathrm{L} / \mathrm{M}$ and orexin-A levels between the two groups before surgery and on the 1st postoperative day; those indexes were statistically decreased in the observation as compared to the control group on the 7 th and 14 th postoperative days. The nutritional status grades were evaluated using a Patient-Generated Subjective Global Assessment (PG-SGA) quantitative scoring at 3 months after surgery and showed that malnutrition rates were significantly lower in the observation as compared to the control group. Serum levels of hemoglobin ( $\mathrm{Hb})$, albumin (Alb), prealbumin (PAB) and transferrin (TRF), 3 months after surgery showed significantly lower $\mathrm{Hb}$ and $\mathrm{Alb}$ and significantly higher PAB and TRF levels in the observation compared to the control group. After one year follow-up, there were no significant differences in hospitalization time and cost between the two groups, but the postoperative complication, readmission and mortality rates in the observation group were significantly lower than that the control group. Each dimension score of quality of life in the observation group was significantly higher than the control group. In conclusion, the $\mathrm{H} 2 \mathrm{H}$ nutrition management model can effectively improve the
\end{abstract}

Correspondence to: Dr Haizhen Liu, Department of Clinical Nutrition, Weifang People's Hospital, 151 Guangwen Street, Kuiwen, Weifang, Shandong 261041, P.R. China

E-mail: nk45fn@163.com

Key words: Hospital to Home nutrition management, laryngeal carcinoma, clinical outcomes, orexin-A, L/M, quality of life postoperative nutritional level of patients with laryngeal carcinoma, resulting in a lower rate of complication, readmission and mortality resulting in an improved patient's quality of life.

\section{Introduction}

The function of the larynx includes deglutition, phonation and facilitates respiration. Laryngeal carcinoma is a common malignant tumor that is primarily treated by surgical resection (1). The patient's stress level after surgical resection (in addition to side effects of radiotherapy and chemotherapy) is increased due in part to the close association between the structure and function characteristics of larynx and hosts nutritional status. Patients, while under the body's high metabolic status after surgery, can often develop varying degrees of eating disorders and a relative malnutrition state that can negatively impact the patient's quality of life $(2,3)$. To prevent or reverse the postoperative nutritional problems, attention directed to nutritional support therapies may have a great significance in order to prolong the patient's survival time and reduce the mortality rate (4). A Hospital to Home $(\mathrm{H} 2 \mathrm{H})$ nutrition management model is an individualized nutrition management strategy proposed by the clinical nutrition department in West China Hospital of Sichuan University (Chengdu, China), with the objective to extend nutritional therapy from hospital to discharge and transform a single treatment approach into therapeutic regimen with multiple models. The purpose of this investigation is to determine the effectiveness of a $\mathrm{H} 2 \mathrm{H}$ nutrition management model on the postoperative clinical outcome of patients with surgically treated laryngeal carcinoma.

\section{Materials and methods}

Eighty-six patients with laryngeal carcinoma admitted to Weifang People's Hospital (Shandong, China) from October 2014 to September 2015, were selected for possible study inclusion. Inclusion criteria: Patients with a confirmed diagnosis of laryngeal carcinoma by computed tomography (CT) or magnetic resonance imaging $(\mathrm{MRI})$ who did not receive radiotherapy or chemotherapy before operation; who underwent surgical resection; who had an expected survival period of more than 3 months; and who had signed an informed consent. Patients were excluded if they had abnormal coagulation function, or obnubilation or a communication barrier. Patients were randomly divided (using 
a random number table) into either an observation $(n=43)$ or control group $(n=43)$ and demographic data were recorded.

All the patients were treated with surgery; the nutritional status was comprehensively evaluated before treatment, and an early nutritional screening was conducted. The control group received routine nutritional support therapy after surgery; the nasogastric tube was indwelt before operation and enteral nutritional support was given at $48 \mathrm{~h}$ postoperative; patients were given a gradual increase of Nutrison Fibre (Nutricia Pharmaceutical Co., Ltd., Wuxi, China) by continuous micropumping (pump speed as 50-100 $\mathrm{ml} / \mathrm{h}$ ); nutritional support was given to patients after the caloric requirement was calculated according to $20-30 \mathrm{kcal} /(\mathrm{kg} \cdot$ day $)$ standard within the 1 st to $3 \mathrm{rd}$ day of nutritional therapy, and the total volume was increased to $2,000 \mathrm{ml}$ from the 4th day. Nutrient solution temperature was maintained at $37^{\circ} \mathrm{C}$ by infusion thermostat, and gastric juice was pumped back every $5 \mathrm{~h}$ and observed; when the gastric juice volume was more than $200 \mathrm{ml}$, the pumping was stopped temporarily. The nutrient solution infusion volume was gradually decreased when patients could take food by mouth, and the total calorie intake was no less than $25 \mathrm{kcal} /(\mathrm{kg} \cdot$ day $)$.

The observation group was treated with the $\mathrm{H} 2 \mathrm{H}$ nutrition management model; the individualized therapeutic regimen was formulated according to the results of nutritional screening before surgery: i) Preoperative nutritional support therapy: Nutritional education was performed for patients and their families; for patients with well nutrition or mild malnutrition, glucose and electrolyte infusion were taken before surgery, and for patients with moderate to severe malnutrition, parenteral nutritional support was administered; ii) postoperative nutritional therapy during hospitalization: Early enteral nutritional support was taken, within $24 \mathrm{~h}$ after operation, and the specific methods were the same as those in the control group; and iii) nutrition therapy outside the hospital: Patients were maintained with nutritional support by continuous nutritional therapy until 6 weeks after discharge.

Before surgery, patients orally ingested $2 \mathrm{~g}$ lactulose and $1 \mathrm{~g}$ mannitol on an empty stomach; lactulose and mannitol with the same grams were injected into nutritive canal on the 1st, 7th and 14th postoperative day, and the total urine of patients was collected within $6 \mathrm{~h}$, adding dimethylbenzene, and then storing it at $-20^{\circ} \mathrm{C}$, before analysis. The samples were thawed, centrifuged 3,650 x $\mathrm{g}$ for $5 \mathrm{~min}$; and the protein and ions were removed. To the supernatant was added acetonitrile, mixing uniformly, and then centrifuging at 1,050 x g for $10 \mathrm{~min}$. Protein and ion were removed, and the supernatant was isolated. The lactulose and mannitol were respectively prepared for the samples with concentrations of 1,000, 500, 200,100 and $50 \mathrm{mg} / \mathrm{l}$ by ultrapure water, and detected by the high-performance liquid chromatography differential detection method. The concentration of lactulose and mannitol was calculated in accordance with standard curve computational formula, and the ratio of lactulose to mannitol (L/M) was calculated.

Venous blood $(5 \mathrm{ml})$ was collected before operation and on the 1st, 7th and 14th postoperative day and centrifuged $2,750 \mathrm{x} \mathrm{g}$ for $20 \mathrm{~min}$ for $20 \mathrm{~min}$; supernatant was collected and stored at $-20^{\circ} \mathrm{C}$. The serum orexin-A level of patients was determined by enzyme-linked immunosorbent assay (ELISA),
Table I. Comparison of baseline data of patients between the two groups.

\begin{tabular}{lcccc}
\hline & $\begin{array}{c}\text { Control } \\
\text { group } \\
(\mathrm{n}=43)\end{array}$ & $\begin{array}{c}\text { Observation } \\
\text { group } \\
(\mathrm{n}=43)\end{array}$ & $\mathrm{t} / \chi^{2}$ & P-value \\
\hline $\begin{array}{l}\text { Items } \\
\text { Sex }\end{array}$ & $24 / 19$ & $21 / 22$ & 0.420 & 0.517 \\
$\begin{array}{l}\text { Age (years) } \\
\text { Average }\end{array}$ & $45-76$ & $45-75$ & & \\
age (years) & $55.76 \pm 6.43$ & $56.03 \pm 6.56$ & 0.193 & 0.847 \\
$\begin{array}{l}\text { Operation } \\
\text { time (min) }\end{array}$ & $203.54 \pm 61.17$ & $204.43 \pm 61.36$ & 0.067 & 0.947 \\
$\begin{array}{l}\text { Tumor staging } \\
\text { (n, \%) }\end{array}$ & & & & \\
$\begin{array}{l}\text { I-II } \\
\text { III-IV }\end{array}$ & $23(53.49)$ & $21(48.84)$ & 0.186 & 0.666 \\
$\begin{array}{l}\text { Operation } \\
\text { mode (n, \%) } \\
\begin{array}{l}\text { Partial } \\
\text { laryngectomy }\end{array}\end{array}$ & $20(46.51)$ & $22(51.16)$ & 0.186 & 0.666 \\
$\begin{array}{l}\text { Total } \\
\text { laryngectomy }\end{array}$ & $17(39.53)$ & $15(34.88)$ & 0.199 & 0.655 \\
\hline & $26(60.47)$ & $28(63.12)$ & 0.199 & 0.655 \\
\hline & & & \\
\hline
\end{tabular}

using kits (Shanghai Jiang Lai Biotechnology Co., Ltd., Shanghai, China) according to the manufacturer's instructions. At postoperative 3 months, $10 \mathrm{ml}$ fasting peripheral venous blood was used to determine levels of hemoglobin $(\mathrm{Hb})$, albumin (Alb), prealbumin (PAB) and transferrin (TRF) using a fully automatic biochemical analyzer.

All patients were followed-up for 1 year, and the readmission rates and mortality rates were recorded; all patients were regularly re-examined in the out-patient department; patients in the observation group were followed up by WeChat network platform, mobile APP, telephone and family visit. A home visit was performed for patients treated with home parenteral nutritional support by community physicians every week.

The total urine was collected within $6 \mathrm{~h}$ after patients orally ingested the lactulose and mannitol before operation and on the 1st, 7th and 14th postoperative day, and L/M was detected by the high-performance liquid chromatography differential detection method and recorded; $5 \mathrm{ml}$ venous blood was analyzed before surgery and on the 1st, 7th and 14th postoperative day and serum orexin-A level of patients was determined by ELISA.

At postoperative 3 months, $10 \mathrm{ml}$ fasting peripheral venous blood was analyzed for levels of $\mathrm{Hb}, \mathrm{Alb}, \mathrm{PAB}$ and TRF using a fully automatic biochemical analyzer. The nutritional status of patients in the two groups was evaluated by PG-SGA quantitation, and the grade standards were: i) Good nutrition, PG-SGA score $\leq 1$ point; ii) mild/moderate malnutrition, 2 points $\leq$ PG-SGA score $\leq 8$ points; and iii) severe malnutrition, PG-SGA score $\geq 9$ points.

At postoperative 6 months, the quality of life of patients was assessed by Quality of Life Questionnaire-Core 30 (QLQ-C30) (5), and the scoring was respectively completed from 5 dimensions, including body function, role function, 


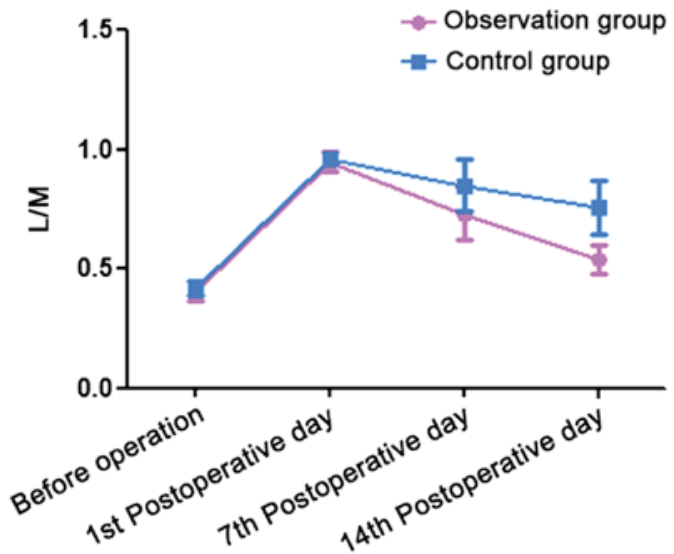

Figure 1. L/M values of patients in the two groups. The $\mathrm{L} / \mathrm{M}$ value in each group before operation is compared with that on the 1st, 7th and 14th postoperative day $(\mathrm{p}<0.05) ; \mathrm{L} / \mathrm{M}$ in the observation group is compared with that in the control group before operation and on the 1st postoperative day $(\mathrm{p}>0.05)$; $\mathrm{L} / \mathrm{M}$ in the observation group is compared with that in the control group on the 7 th and 14 th postoperative day $(\mathrm{p}<0.05)$. L/M, lactulose to mannitol.

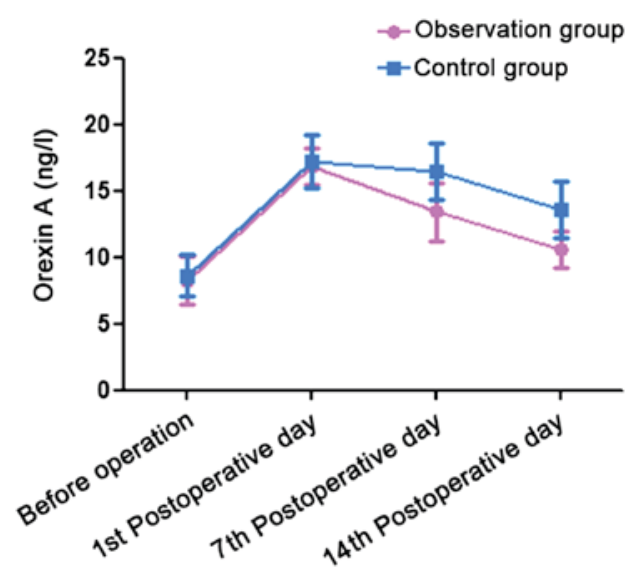

Figure 2. Orexin-A levels of patients in the two groups. The orexin-A level in each group before operation is compared with that on the 1st, 7th and 14th postoperative day $(\mathrm{p}<0.05)$; orexin-A level in the observation group is compared with that in the control group before operation and on the 1st postoperative day ( $>0.05)$; orexin-A level in the observation group is compared with that in the control group on the 7 th and 14 th postoperative day $(\mathrm{p}<0.05)$.

cognitive function, emotional function and social function; the original score was converted into centesimal system by linear formula; the higher score correlated to a higher quality of life.

The hospitalization time, hospitalization cost and incidence rate of postoperative complication of patients were determine after the one year follow-up and the readmission rate and mortality rate of patients in the two groups were compared.

Data were processed using SPSS 19.0 (SPSS, Inc., Chicago, IL, USA). Measurements were expressed as mean \pm standard deviation (mean $\pm \mathrm{SD}$ ), and t-test was utilized; enumeration data were expressed by ratio and Chi-square test was utilized. $\mathrm{P}<0.05$ was considered to indicate a statistically significant difference.

\section{Results}

The demographic data of patients between the two groups was not statistically different $(\mathrm{p}>0.05)$ (Table I). Before
Table II. Comparison of postoperative serum nutritional indexes of patients between the two groups.

\begin{tabular}{lccccc}
\hline Groups & Case & $\begin{array}{c}\mathrm{Hb} \\
(\mathrm{g} / \mathrm{l})\end{array}$ & $\begin{array}{c}\text { Alb } \\
(\mathrm{g} / \mathrm{l})\end{array}$ & $\begin{array}{c}\mathrm{PAB} \\
(\mathrm{g} / \mathrm{l})\end{array}$ & $\begin{array}{c}\mathrm{TRF} \\
(\mathrm{g} / \mathrm{l})\end{array}$ \\
\hline Observation & 43 & $93.43 \pm 5.38$ & $38.52 \pm 3.59$ & $0.26 \pm 0.09$ & $1.76 \pm 0.34$ \\
Control & 43 & $96.57 \pm 5.76$ & $41.41 \pm 3.63$ & $0.18 \pm 0.06$ & $1.38 \pm 0.47$ \\
t-test & & 2.612 & 3.712 & 4.850 & 4.296 \\
P-value & & 0.011 & $<0.001$ & $<0.001$ & $<0.001$ \\
\hline
\end{tabular}

$\mathrm{Hb}$, hemoglobin; Alb, albumin; PAB, prealbumin; TRF, transferrin.

Table III. Therapeutic effects of patients in the two groups.

\begin{tabular}{lcccc}
\hline Groups & Case & $\begin{array}{c}\text { Hospitalization } \\
\text { time } \\
\text { (days) }\end{array}$ & $\begin{array}{c}\text { Hospitalization } \\
\text { cost } \\
\text { (thousand RMB) }\end{array}$ & $\begin{array}{c}\text { Postoperative } \\
\text { complication } \\
\text { (n, \%) }\end{array}$ \\
\hline Observation & 43 & $11.82 \pm 3.23$ & $2.73 \pm 1.24$ & $3(6.98)$ \\
Control & 43 & $12.14 \pm 3.24$ & $2.65 \pm 1.38$ & $12(27.91)$ \\
$\mathrm{t} / \chi^{2}$ & & 0.459 & 0.283 & 5.174 \\
P-value & & 0.647 & 0.778 & 0.023 \\
\hline
\end{tabular}

Table IV. Comparison of adverse reactions of patients between the two groups $(\mathrm{n}, \%)$.

\begin{tabular}{lccc}
\hline Groups & Case & Readmission rate & Mortality rate \\
\hline Observation & 43 & $4(9.30)$ & $3(6.98)$ \\
Control & 43 & $15(34.88)$ & $13(30.23)$ \\
$\chi^{2}$ & & 6.756 & 6.223 \\
P-value & & 0.009 & 0.012 \\
\hline
\end{tabular}

operation, and on the 1st, 7th and 14th postoperative day, L/M in the observation group were $0.41 \pm 0.12,0.95 \pm 0.23,0.73 \pm 0.14$ and $0.54 \pm 0.13$, respectively, and were $0.42 \pm 0.11,0.96 \pm 0.24$, $0.85 \pm 0.15$ and $0.76 \pm 0.14$ in the control group; before operation and on postoperative 1st, 7th and 14th day, orexin-A levels in the observation group were $8.32 \pm 2.14,16.86 \pm 4.28,13.47 \pm 3.26$ and $10.63 \pm 1.08 \mathrm{ng} / 1$, respectively, and were $8.67 \pm 2.34$, $17.24 \pm 4.35,16.49 \pm 3.42$ and $13.63 \pm 1.39 \mathrm{ng} / 1$ in the control group; L/M and orexin-A levels of patients in the two groups on the 1st postoperative day were significantly higher than those before operation, which were decreased from the 7th and 14th postoperative day, but higher than those before operation $(\mathrm{p}<0.05)$; there were no statistical differences between the two groups before operation and on the 1st postoperative day $(p>0.05)$, and the indexes in the observation group were significantly decreased than those in the control group on the 7th and 14th postoperative day (Figs. 1 and 2).

The serum Hb, Alb, TRF and PAB of patients between the two groups at 3 months postoperative were compared (Table II). $\mathrm{Alb}$ and $\mathrm{Hb}$ of patients in the observation group were significantly lower $(\mathrm{p}<0.05)$ than those in the control group, the levels of TRF and PAB in the observation group were significantly higher $(\mathrm{p}<0.05)$ than those in the control group $(\mathrm{p}<0.05)$. 


\section{The nutritional status of patients was determined using PG-SGA quantitation}

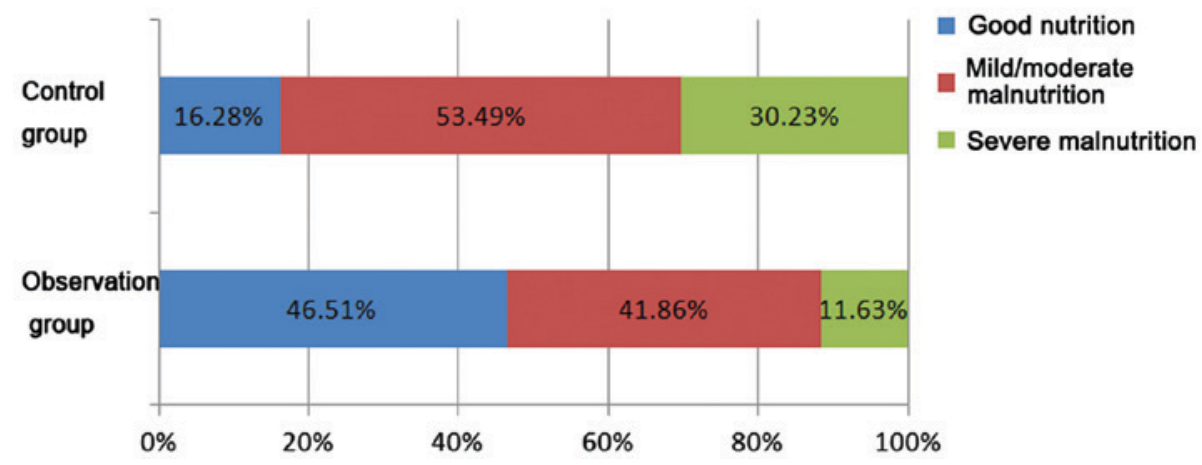

Figure 3. Evaluating nutritional status of patients by PG-SGA quantitation; comparisons between the two groups, $\mathrm{p}<0.05$. PG-SGA, Patient-Generated Subjective Global Assessment.

Table V. Comparisons of quality of life of patients between the two groups.

\begin{tabular}{lcccccc}
\hline Groups & Case & Body function & Role function & Cognitive function & Emotional function & Emotional function \\
\hline Observation & 40 & $91.62 \pm 3.23$ & $92.73 \pm 1.24$ & $88.72 \pm 3.23$ & $72.73 \pm 3.24$ & $87.73 \pm 5.44$ \\
Control & 30 & $83.74 \pm 3.24$ & $84.65 \pm 1.38$ & $80.34 \pm 3.24$ & $64.65 \pm 3.38$ & $82.65 \pm 6.38$ \\
$\mathrm{t} / \chi^{2}$ & & 10.088 & 25.704 & 10.728 & 10.136 & 3.590 \\
P-value & $<0.001$ & $<0.001$ & $<0.001$ & 0.001 & 0.001 \\
\hline
\end{tabular}

The nutritional status of patients was determined using PG-SGA quantitation (Fig. 3). The mild/moderate and severe malnutrition rates in the observation group were $41.86 \%(18 / 43)$ and $11.63 \%$ (5/43), respectively, which were $53.49 \%(23 / 43)$ and $30.23 \%$ (13/43) in the control group; the malnutrition rates were significantly lower in the observation group than those in the control group $(\mathrm{p}<0.05)$.

The incidence rate of postoperative complication, hospitalization time and hospitalization cost of patients in the two groups were compared (Table III). There were no statistical differences in comparison of hospitalization time and hospitalization cost of patients between the two groups ( $p>0.05)$, and the incidence rate of postoperative complication in the observation group was significantly lower than that in the control group $(\mathrm{p}<0.05)$.

The readmission rate and mortality rate of patients in the two groups after one-year follow-up were compared (Table IV). The readmission rate and mortality rate in the observation group $(9.30$ and $6.97 \%)$ were significantly lower $(\mathrm{p}<0.05)$ than the control group (34.88 and 30.23\%).

The quality of life of patients between the two groups after one-year follow-up (deceased patients were excluded) were compared (Table V). Each dimension score of quality of life in the observation group was significantly higher $(\mathrm{p}<0.05)$ than the control group.

\section{Discussion}

Laryngeal carcinoma is one of the most common malignant tumors in otorhinolaryngology head and neck surgery, accounting for 1 to $5 \%$ of systemic tumor, and its incidence rate is increasing (6). Approximately, 30 to $90 \%$ of cancer patients will be malnourished (the malnutrition rate in patients with laryngeal carcinoma is higher than in those with other cancers) and $20 \%$ of patients with cancer will be deceased due to malnutrition and related complications (7). Multiple factors can lead to malnutrition in patients with laryngeal carcinoma, including therapy, tumor load and psychological factors (8). Generally, patients with laryngeal carcinoma cannot eat food by mouth for about two weeks after surgery. From surgery, the integrity of laryngeal physiological function can be damaged which can cause partial or total loss of its protective effects, leading to dysphagia. In addition, the host caloric needs can be increased by blood loss during and after surgery, pain and other stress responses, resulting in a loss of appetite, gastrointestinal symptoms and oral infections. These factors of an increased caloric need and a decreased dietary caloric intake can lead to weight loss and reduced subcutaneous fat, thus inducing or aggravating malnutrition $(9,10)$.

Nutritional oncology is an area of combined nutrition theory and methods for the prevention (can accurately evaluate nutritional status of patients with cancer to diagnose malnutrition early in the disease/treatment process) and treatment (provide appropriate nutritional support therapy) of cancer patients) (11). Most of the traditional nutritional therapies focus on the nutritional status of patients only during hospitalization after surgery, and often neglect the nutritional status of the patients outside the hospital. After discharge of patients with laryngeal carcinoma, anorexia, nausea, vomiting and other symptoms often occur after postoperative radiotherapy and chemotherapy, and in particular, metabolic disorders caused by cachexia can appear in patients with advanced laryngeal carcinoma; therefore, it is necessary to improve the nutrition maintenance therapy of patients once outside 
the hospital (12-14). The $\mathrm{H} 2 \mathrm{H}$ nutrition management model is a comprehensive treatment model with the patient at the center with continuous nutrition therapy for that patient from hospitalization to outside the hospital; the model uses a team approach involved by many medical disciplines (specialist physician, nutritionist, community physician, nurse and patient's family), which can improve patient compliance with nutritional therapy and minimize or ameliorate the status of malnutrition in patients. The results of this study showed the readmission and mortality rate in the observation group were distinctly lower than those in the control group $(\mathrm{p}<0.05)$, which demonstrates the validity of improved patient outcomes with the implementation of the $\mathrm{H} 2 \mathrm{H}$ nutrition management model.

The results of this study showed that orexin-A levels and $\mathrm{L} / \mathrm{M}$ of patients in the two groups on the 1st postoperative day were significantly higher than those before operation, and decreased from postoperative 7 th and 14th day, but higher than those before operation $(\mathrm{p}<0.05)$; there were no statistical differences between the two groups before operation and on postoperative 1st day ( $p>0.05$ ), and the indexes in the observation group were significantly decreased over the control group on the 7 th and 14 th postoperative day $(\mathrm{p}<0.05)$; the increase of $\mathrm{L} / \mathrm{M}$ reveals that the tight junction of intestinal mucosa is incomplete, permeability is increased, and intestinal barrier function is damaged $(15,16)$; the possible causes include: i) Intestinal secretory immunoglobulin A content in patients treated by surgery is decreased; ii) intestinal colonization resistance is reduced and iii) the normal intestinal function is inhibited. Many inflammatory mediators can be activated by the stress response of surgical trauma; for example the gastrointestinal mucosal barrier can be damaged by interleukin (IL) and tumor necrosis factor- $\alpha$ (TNF- $\alpha$ ) and an excess amount of NO production, that is induced by TNF- $\alpha$, can cause microcirculation congestion resulting in damage to the integrity of intestinal membrane barrier (17). Early enteral nutritional support during hospitalization, and continuous parenteral nutritional support after discharge, can provide the necessary fuel for the host to repair the injury of intestinal epithelium and effectively protect the chemical barrier of intestinal mucosa; this recovery of the intestinal barrier function is gradual and aids in amelioration of the patients' malnourished status (18). Orexin-A, is a neuropeptide that can regulate the body's energy metabolism and improve appetite (14). The related experiment reveals that neuron of appetite function can be inhibited by orexin-A, which can excite hypothalamic NPY/cocaine- and amphetamine-regulated transcript peptide neuron, thus promoting the increase of body's food intake and weight (15). The increased expression level of orexin-A caused by postoperative fasting will promote the patient's appetite and with the support of postoperative enteral nutrition, especially early nutritional support, food intake is increased and the body's energy metabolism is improved, thereby gradually downregulating orexin-A expression to the normal level.

The results of this study indicated that the incidence rate of postoperative complication in the observation group was significantly lower than that in the control group $(\mathrm{p}<0.05)$. It appears that early enteral nutritional support can effectively reduce bacterial endotoxin translocation, stimulate secretion of digestive juice and gastrointestinal hormones, promote normal gastrointestinal peristalsis, and effectively avoid severe consumption of high catabolism caused by prolonged postoperative fasting, allowing the host metabolic state to return to the normal (early recovery of preoperative nutritional status and improved body's negative nitrogen balance) and prevent the occurrence of postoperative complications caused by malnutrition (19). Results from this investigation noted that between the two groups, serum Alb and $\mathrm{Hb}$ of patients in the observation group were significantly lower than those in the control group, and the levels of TRF and PAB in the observation group were significantly higher than the control group, suggesting that the nutritional level of patients intervened by the $\mathrm{H} 2 \mathrm{H}$ nutrition management model is significantly ameliorated, thus reducing the incidence rate of malnutrition.

Currently, PG-SGA evaluation is utilized for patients with laryngeal carcinoma to screen for malnutrition risk. Advantages of this tool is that it can quantitatively evaluate patient's nutritional status from measurement of upper limb muscle circumference, triceps skinfold thickness, maximum circumferential diameter of crus, grip strength of non-handedness and body mass index (BMI) (20). By adopting the $\mathrm{H} 2 \mathrm{H}$ nutrition management model, PG-SGA evaluation showed that malnutrition rates were significantly lower in the observation group than the control group, and each dimension score of quality of life in the observation group was distinctly higher than the control group ( $<<0.05)$. It should be emphasized that the nutritional therapy of patients administered by the $\mathrm{H} 2 \mathrm{H}$ nutrition management model is not static after hospital discharge; a continuous and individualized nutritional support therapy is implemented for patients through PG-SGA evaluation; the follow-up is regularly conducted, and the intake, body weight change, and effect of nutritional support therapy, of patients are observed; in addition, effective psychological counseling is also important to manage the patients with depression, self-abasement and other social barriers, in order to improve patients' quality of life.

In conclusion, the $\mathrm{H} 2 \mathrm{H}$ nutrition management model can ameliorate the postoperative malnutritional status of patients with laryngeal carcinoma, reduce the incidence rate of malnutrition and postoperative complication and improve the postoperative quality of life of patients, thus promoting an effective and rational utilization of medical resources.

\section{References}

1. Li L, Xu Y and Wang B: Liriodenine induces the apoptosis of human laryngocarcinoma cells via the upregulation of p53 expression. Oncol Lett 9: 1121-1127, 2015.

2. Li P, Zhang J, Sun G and Li H: Assessment of quality of life for laryngeal cancer patients after laryngectomy. Lin Chung Er Bi Yan Hou Tou Jing Wai Ke Za Zhi 28: 1648-1651, 2014 (In Chinese).

3. Kaya E, Cingi C, Pınarbaşlı Ö, Gürbüz MK, Çaklı H, İncesulu A, Özüdoğru E and Keçik C: Health-related quality of life in laryngeal cancer patients. J Med Updates 4: 29-36, 2014.

4. Shivappa N, Hébert JR, Polesel J, Zucchetto A, Crispo A, Montella M, Franceschi S, Rossi M, La Vecchia C and Serraino D: Inflammatory potential of diet and risk for hepatocellular cancer in a case-control study from Italy. Br J Nutr 115: 324-331, 2016.

5. Bedard G, Zeng L, Zhang L, Lauzon N, Holden L, Tsao M Danjoux C, Barnes E, Sahgal A, Poon M, et al: Minimal important differences in the EORTC QLQ-C30 in patients with advanced cancer. Asia Pac J Clin Oncol 10: 109-117, 2014.

6. Agra IM, Ferlito A, Takes RP, Silver CE, Olsen KD, Stoeckli SJ, Strojan P, Rodrigo JP, Gonçalves Filho J, Genden EM, et al: Diagnosis and treatment of recurrent laryngeal cancer following initial nonsurgical therapy. Head Neck 34: 727-735, 2012. 
7. Chen Y, Liu BL, Shang B, Chen AS, Liu SQ, Sun W, Yin HZ, Yin JQ and Su Q: Nutrition support in surgical patients with colorectal cancer. World J Gastroenterol 17: 1779-1786, 2011.

8. Shpata V, Prendushi X, Kreka M, Kola I, Kurti F and Ohri I: Malnutrition at the time of surgery affects negatively the clinical outcome of critically ill patients with gastrointestinal cancer. Med Arch 68: 263-267, 2014.

9. Lu W, Xiong N, Lyu Z and Xu W: Effect of peer education on postoperative rehabilitation of patients with laryngeal cancer. Zhonghua Er Bi Yan Hou Tou Jing Wai Ke Za Zhi 49 632-636, 2014 (In Chinese).

10. Langius J A E, Twisk J, Kampman M, Doornaert P, Kramer MH Weijs PJ and Leemans CR: Prediction model to predict critical weight loss in patients with head and neck cancer during (chemo) radiotherapy. Oral Oncol 52: 91-96, 2016.

11. Omlin A, Blum D, Wierecky J, Haile SR, Ottery FD and Strasser F: Nutrition impact symptoms in advanced cancer patients: Frequency and specific interventions, a case-control study. J Cachexia Sarcopenia Muscle 4: 55-61, 2013.

12. Skóra T, Nowak-Sadzikowska J, Mucha-Małecka A, SzyszkaCharewicz B, Jakubowicz J and Gliński B: Postoperative irradiation in patients with pT3-4N0 laryngeal cancer: Results and prognostic factors. Eur Arch Otorhinolaryngol 272: 673-679, 2015.

13. Cotogni P: Enteral versus parenteral nutrition in cancer patients: Evidences and controversies. Ann Palliat Med 5: 42-49, 2016.

14. Leonard CS and Kukkonen JP: Orexin/hypocretin receptor signalling: A functional perspective. Br J Pharmacol 171: 294-313, 2014
15. Messina G, Dalia C, Tafuri D, Monda V, Palmieri F, Dato A, Russo A, De Blasio S, Messina A, De Luca V, et al: Orexin-A controls sympathetic activity and eating behavior. Front Psychol 5: 997, 2014.

16. Earley ZM, Akhtar S, Green SJ, Naqib A, Khan O, Cannon AR, Hammer AM, Morris NL, Li X, Eberhardt JM, et al: Burn injury alters the intestinal microbiome and increases gut permeability and bacterial translocation. PLoS One 10: e0129996, 2015.

17. Siplivy $\breve{~ V A, ~ G r i n c h e n k o ~ S V, ~ G o r g o l ' ~ N I, ~ D o t s e n k o ~ V V ~ a n d ~}$ Evtushenko AV: Pathomorphological peculiarities of hemomicrocirculatory bed of the small and large intestine in acute peritoniti. Klin Khirurgiia 29: 61-63, 2014.

18. Xu XD, Sun YS, Shao QS, Hu JF, Qian ZY, Zhou YL and Ye ZY: Effect of early enteral nutrition supplemented with glutamine on postoperative intestinal mucosal barrier function in patients with gastric carcinoma. Zhonghua Wei Chang Wai Ke Za Zhi 14: 436-439, 2011 (In Chinese).

19. Li NM, Liu F, Lv FY and Zhang QW: Influencing factors and interventional strategies for early enteral nutrition after gastric carcinoma surgery. J Cancer Res Ther 12: 689-692, 2016.

20. Um MH, Choi MY, Lee SM, Lee IJ, Lee CG and Park YK Intensive nutritional counseling improves PG-SGA scores and nutritional symptoms during and after radiotherapy in Korean cancer patients. Support Care Cancer 22: 2997-3005, 2014. 